\title{
I think I understand: Investigating misconceptions regarding hypothesis test concepts among university students
}

\author{
Aida Zaini \\ Universiti Brunei Darussalam \\ Haziq Jamil* \\ Universiti Brunei Darussalam \\ Elvynna Leong \\ Universiti Brunei Darussalam
}

9 September 2021

\begin{abstract}
Hypothesis testing is an essential tool among researchers and practitioners alike, with its use being being widely taught in many a programme at university level. However, past studies have shown that students hold misconceptions about important statistical concepts. This present study aims to reconfirm past efforts in this area, specifically in a South East Asian higher education institution. To test how well undergraduate university students' understood key concepts in hypothesis testing, an online multiple choice questionnaire was deployed. The questionnaire also asked for students' confidence ratings for each question, allowing us to distinguish the confident versus non-confident incorrect responses. A followup interview was then conducted to give deeper insights into reasons behind respondents' errors. The main finding is that there are significantly more confident wrong answers than non-confident ones - highly indicative of the presence of misconceptions among respondents. Among them, students firmly believed that statistical inference procedures provide a direct calculational proof of the null hypothesis. Additionally, students have difficulty formulating correct hypotheses to be tested, and have poor grasp of the role of significance levels in hypothesis testing. Whether or not students were taking a quantitative-focused programme, or had prior statistics training, had no bearing on their score. Despite this, confidence ratings were significantly higher in both groups.
\end{abstract}

Keywords: Statistics education; Hypothesis testing; Misconceptions; Undergraduate students; Confidence

\section{Introduction}

The use of statistics is seen widely to conduct research and make decisions effectively across a variety of fields such as education, business, healthcare, and others. There are broadly two halves to the term 'statistics', with one being descriptive statistics such as calculating means and medians; and the other statistical inference (which includes the topic of interest, hypothesis testing). Mastery of the latter is essential to any form of quantitative work, especially when outcomes revolve around assessing what the data say about the plausibility of a specific research hypothesis.

In Brunei Darussalam, students are expected to encounter introductory statistics as early as high school. Statistics topics are examined as early as in Cambridge General Certificate of Education Ordinary Level (GCE O-Level) examinations in high school, and then further in GCE

\footnotetext{
${ }^{*}$ Corresponding author. E-mail $\sqrt{\text { haziq.jamil@ubd.edu.bn }}$
} 
Advanced Level (GCE A-Level) examinations prior to university. For example, in GCE O-Level Statistics, one of the syllabi aims is for learners to be able to "interpret both primary statistical information and the conclusions of statistical analysis" (Cambridge Assessment International Education, 2018). Whereas in the GCE A-Levels, one of the aims in the Statistics section is to be able to "understand the nature of a hypothesis test and the terms null hypothesis, alternative hypothesis, significance level, rejection region (or critical region), acceptance region and test statistic" (Cambridge Assessment International Education, 2020). In Brunei Darussalam especially, there is a high motivation for students to take STEM-based subjects as much as possible (Sekretariat Tetap Wawasan Brunei 2035, 2020), to the point where mathematics is deemed almost compulsory, especially when aspirations for further studies exist. Thus, it would be a fair statement to say that students entering university in Brunei Darussalam will have some level of familiarity of basic statistical concepts.

At Universiti Brunei Darussalam (UBD), statistics is taught both in a primary role (e.g. at the Mathematics Department in the Faculty of Science), and in a supporting role (i.e. as standalone modules in a wider programme e.g. in Health Science, Business and Economics, Education, etc.). Due to the sheer number of students, it is not possible to have a shared "service-level" module across all faculties, and as a result, each faculty is in complete control of their statistics courses. Understandably the courses are tailored to suit individual programmes, so there is expected to be an interesting variation in the teaching methods. As a for instance, Mathematics majors do tend to focus more on the mathematical theories of random events and statistical inference, whereas those enrolled outside of Mathematics will tend to focus on the practical aspects of statistical inference, including the use of computer software. One thing in common for sure is an emphasis on hypothesis testing, regardless of teaching faculty. But does the variation in teaching methods and learning emphases impact understanding of hypothesis test concepts?

Numerous studies have shown that students have misconceptions on statistical inference for the past three decades (Burns-Childers et al., 2017; Mevarech, 1983; Smith, 2008; Sotos et al., 2007, 2009, Vallecillos, 2000). In the current day and age of data, familiarity with statistical techniques is not just beneficial to students from a post-education and job market perspective, it also provides the numerical literacy to navigate the complex, information-centric world we currently live in. There is definitely a case for students to grasp the ideas of statistical reasoning and literacy, and conceptual understanding in statistics that is often overlooked in favour of results-oriented outputs. Having said this, hypothesis testing has been expressed as one of the most misunderstood topics in statistics (Vallecillos, 2000). Due to such rampant misconceptions, students are generally weaker in using hypothesis test tools to interpret data compared to direct numerical calculations (Nickerson, 2000) in order to make meaningful worldview interpretations. It is also reported (Batanero, 2000) that there are three major aspects of the statistical hypothesis that are found to be difficult for students, namely, to understand the concept of a hypothesis test, to interpret p-values and to interpret the significance level. These are certainly the topics that we aim to investigate in our own study. One interest would be in seeing whether or not there is any correlation between academic performance and having misconceptions. More broadly, what factors make it so that statistics students become more prone to misconceptions?

With this in mind, the primary goal of this study was to establish first and foremost whether misconceptions do exist among undergraduate university students studying statistics. Furthermore, we also explore the following research questions:

1. What factors affect having misconceptions in hypothesis testing?

2. What are the most common errors made in hypothesis testing among students?

3. What are the reasons that such errors are made? 


\section{Literature review}

A review of the literature since the 1980s suggests that students of statistics do have trouble with understanding hypothesis testing concepts (Burns-Childers et al., 2017; Mevarech, 1983; Smith, 2008; Sotos et al., 2007, 2009: Vallecillos, 2000). The summary of the issue at hand here is a failure of students to comprehend the "big picture" of statistical inference, despite having procedural mastery of statistical techniques.

\subsection{Misconceptions in statistical learning}

Researchers agree that even when students are able to perform all the steps of a hypothesis test, misconceptions still occur amongst students as they were unable to explain the reasoning behind these steps. This can be seen from Burns-Childers et al.'s (2017) study that tested students' understanding in test statistics, in which they stated that many students used a procedural approach to hypothesis testing which includes students plugging in correct values in a formula, but ultimately failed to fully understand the real concept. Several of these studies even investigated students' knowledge of introductory statistics among undergraduate students with various education background. Smith (2008) found that well-performing students academically do not necessarily have a solid overall understanding of statistical hypothesis testing. This, in our view, seems to be symptomatic of a highly mechanistic and outcome-oriented style of instruction.

According to Batanero (2000), the common misconceptions revolve around the significance level, interpreting p-values and the role of the null and alternative hypotheses. This leads to several researchers to investigate further into these three aspects of hypothesis testing. Sotos et al. (2009) identified that first year university undergraduates who took introductory statistics are more confident in the hypothesis testing concept but showed significantly lower confidence in interpreting significance levels. Besides this, students tend to not appreciate the role of indirect reasoning and inference in the entire statistical testing paradigm (Smith, 2008). In their study among tertiary education students, Mathews and Clark (2003) and Clark et al. (2007) found that despite great academic achievements, students tend to form misshapen views about learned statistical concepts (namely that of means, standard deviations, and the Central Limit Theorem) six weeks after the completion of their statistics courses. This paragraph does make for bleak reading, especially from the point of view of statistics educations. Incidentally, a small scale study by Liu (2005) managed to find that even educators themselves are prone to confusion regarding common statistical concepts.

\subsection{Diagnostic instruments}

To uncover misconceptions among students learning, an appropriate assessment tool is required. Many formats of these so-called diagnostic tests exist, ranging from open-ended tests to multiple choice questions (MCQs) (Gurel et al., 2015). Pros and cons of both are apparent, but studies seem to favour the use of MCQs (Smith, 2008; Vallecillos \& Batanero, 1997; Vallecillos, 2000), especially using a multi-tier approach. For instance, a two-tier questionnaire would be one in which the first tier consists of conventional knowledge-based questions posed to students, and the second tier then probes for the reasoning behind students' choices (Treagust, 1988). This provides a better way of evaluating students' conceptions - a correct response to the survey item is recorded only if both the answer to the question and the accompanying reason are correct. Note that reasons are typically chosen from a set of multiple choices, or students are prompted for an open-ended reason. Beyond two-tier approaches, three-tier approaches exist as well, with the additional third tier measuring students' confidence in their answers (Arslan et al., 2012, Kirbulut \& Geban, 2014). 
Insofar as obtaining in-depth explanations for diagnosing misconceptions, interviews seem to be the gold standard (Osborne \& Gilbert, 1980). Proponents of this method argue that interviews are the only possible way for students to properly elaborate their cognitive reasoning for answer choices and uncover biases in students' understandings. This was the approach of Kaplan (2009) in their study. Of course, interviews can be integrated with quantitative techniques in a mixed-method approach (Johnson \& Onwuegbuzie, 2004).

As for the actual questions posed, the common approach among diagnostic studies of misconceptions seem to be the design of questions which test statistical reasoning (as opposed to statistical procedures). For instance, Lee and Meletiou (2003) posed a series of questions focusing on investigating levels of understanding regarding descriptive statistics. Questions were more along the lines of "Which of the two histograms show higher variability?" rather than "Construct a histogram from the data provided". In a study by Ismail and Chan (2015), statistical reasoning questions were again posed to investigate misconceptions on the topic of central tendencies.

Hitting a little closer to home, these studies employed statistical reasoning questions that were not typically used under exam conditions: Krishnan and Idris (2014), Rossman and Chance (2014), Smith (2008), and Sotos et al. (2009). In Smith (2008) specifically, a framework for assessing the understanding of statistical hypothesis testing was developed. Each question that was designed for the purpose of their study the fulfils some category and process in the framework. This resulted in a 14-point survey item testing a wide range of knowledge domains. Examples of knowledge domains are the following: recognise applicability, collect a sample, analysis of sample, conclusion, and implication for practice.

\section{Methods}

This study was conducted using a two-phase, mixed method approach of data collection and analysis. The quantitative phase was conducted by disseminating a self-administered online survey designed to test students' statistical reasoning and obtain confidence ratings of their answers. A qualitative phase then ensued in the form of follow-up interviews.

\subsection{Sampling and eligibility}

The study was conducted at Universiti Brunei Darussalam (UBD), situated at Jalan Tungku in Brunei Darussalam. The target population are undergraduate-level students across all faculties and disciplines with familiarity in hypothesis test concepts. A purposive and time-limited sample was obtained so as to obtain a respectable sample size when sliced through certain demographic cross-sections, for the following reasons: It is not known exactly what fraction of the undergraduate students have basic knowledge in hypothesis test concepts. Therefore, a probability sample would have been challenging to implement. Furthermore, response rates was anticipated to be low, judging by the seemingly unpopularity of the study topic.

Note that a range of familiarity levels regarding hypothesis test concepts were entertained, as even those without formal training in statistics were deemed eligible for this study. Here, we define 'formal training' as having taken a statistics-focused class and been assessed for it (e.g. GCE A-Level Statistics or some introductory statistics course at university). Those without formal training had actually encountered hypothesis testing before in situations where statistics was not the main part of the syllabus (e.g. a brief encounter of statistical methods in their respective psychology or biology courses). At the outset, our intention was to distinguish between those with and without formal training and compare the presence and/or persistence of misconceptions in each group. 


\subsection{Instrument}

In order to measure misconception in statistical hypothesis test learning, a two-tier survey was employed. The first tier comprises of a set of five multiple-choice questions designed to measure students' understanding of hypothesis testing. These questions were selected from past studies in this area, namely from the studies of Sotos et al. (2009) and Smith (2008). Therefore, each wrong answer indicates a misconception that might be held by participants regarding the topic. Each of the five survey items can be classified into four categories of hypothesis test knowledge domains, as shown in Table 1 .

Table 1: Categorisation of knowledge domains for the five survey questions.

\begin{tabular}{|c|c|c|}
\hline No & Category & Question \\
\hline 1 & Recognise Applicability & $\begin{array}{l}\text { Which of the following is most likely to be answered } \\
\text { by a study that requires statistical hypothesis testing? }\end{array}$ \\
\hline 2 & Recognise Applicability & $\begin{array}{l}\text { Which of the following statements is the best justi- } \\
\text { fication for using a statistical hypothesis test to an- } \\
\text { swer the question: Are female students as successful } \\
\text { as male students in college mathematics? }\end{array}$ \\
\hline 3 & Generate Hypothesis & $\begin{array}{l}\text { A researcher would like to establish that humani- } \\
\text { ties students have higher GPA than science students. } \\
\text { Which of the following null hypotheses should be } \\
\text { tested? }\end{array}$ \\
\hline 4 & Test Conclusion & $\begin{array}{l}\text { A study tested the claim that: 'Transfer students } \\
\text { are less successful than students admitted as first- } \\
\text { time freshmen'. Results showed a difference in first } \\
\text { semester GPA that is significant at the } 0.05 \text { level. In- } \\
\text { formation from samples is shown below. What is the } \\
\text { most reasonable inference about the population of all } \\
\text { first semester students that can be drawn from this } \\
\text { information? }\end{array}$ \\
\hline 5 & Decision Rule & $\begin{array}{l}\text { To test the hypothesis that private schools are more } \\
\text { effective than public schools, researchers plan to com- } \\
\text { pare the mean starting salaries of private and public } \\
\text { school graduates. But they cannot agree on whether to } \\
\text { test the results at a significance level of } 0.05(\alpha=0.05) \\
\text { or at a significance level of } 0.10(\alpha=0.10) \text {. What ef- } \\
\text { fect will using } 0.10 \text { rather than } 0.05 \text { have on the study? }\end{array}$ \\
\hline
\end{tabular}

Unique to our study was the use of confidence ratings for the second-tier of the questionnaire. Typically, second-tier questions involve respondents' reasoning behind answer choices (Gurel et al., 2015). Since the study involves a qualitative phase as well, confidence ratings give a succinct way of gauging the reliability of respondents' answers. Presumably, we become more ascertained of the accuracy in which respondents' questions reflect their knowledge level given this additional information of confidence ratings.

Therefore, each survey item is accompanied by a 10-point Likert scale rating confidence rating regarding the correctness of respondents' answers (0: Not confident at all; 10: Extremely confident). Such an approach was actually used by Sotos et al. (2009) where the confidence ratings were treated as self-perceived efficacy (Bandura, 1977) in a study primarily investigating confidence of students in their misonceptions about hypothesis tests. With these confidence ratings, it hoped to be able to categorise the respondents according to the categorisations in Table 2 , 
Table 2: Theorised categorisation of respondents broken down by correctness of answers and confidence in answers.

\begin{tabular}{lcc}
\hline & Confident Answer & Non-confident Answer \\
\hline Correct Answer & Good understanding & Poor recall \\
Incorrect Answer & Misconception present & Not knowledgeable \\
\hline
\end{tabular}

\subsection{Demographic variables}

Besides responses to the survey questions and confidence ratings, several demographic information of interest was also collected, namely gender, faculty, programme, year of study, current cumulative grade point average (GPA), and prior statistics experience. Prior experience is defined to be 'Yes' when respondents have had formally studied statistics as a subject either prior to or during university, and 'No' otherwise. We found it more suitable to discretise some of the variables, such as confidence ratings (Not confident: 0-5; Confident: 6-10), GPA (Low: 0-2.5; Medium: 2.5-3.5; High: 3.5-5.0) and programme (Yes: If programme is quantitative in nature; No: Otherwise).

\subsection{Interviews}

In order to obtain deeper insight into students' understanding of hypothesis concepts, a qualitative approach has been made by means of interviews. This qualitative phase of the study would provide information as to 'what' and 'why' the common errors are made i.e the common misconceptions of hypothesis testing. Participants were asked to explain their thought process in answering the multiple-choice questions. At the start of the interview, they were provided with a pen, a sheet of paper and their individual surveys. Interviewees were given two minutes for recall before discussing each item in detail. They were then asked the reason of choosing their answers and/or omitting other options. In addition, they were also allowed to change their answers throughout the interview, should they wish to do so. All interview sessions were recorded and transcribed.

\subsection{Pilot survey}

Prior to actual data collection, an initial pilot survey was deployed and carried out on two volunteers who were final-year undergraduate students majoring in mathematics. The primary focus of this pilot study was to get an approximate completion time, on top of ensuring the appropriate intensity of the survey. The pilot survey was completed in an average of 15 minutes, while the interviews took over an hour.

Originally, ten questions were prepared for the study. However, the pilot survey showed that the two participants were only able to focus on the first seven questions and were then offering answers without proper reasoning on the last three questions due to exhaustion. This also occurred in the follow-up interview as it seemed that participants felt tired when explaining their reasoning after the 45 minutes mark.

In addition, since the original set of ten questions contained multiple questions per knowledge category of interest (refer to Table 1), the two students picked up on this and ended up giving very similar and repetitive explanations for their answers. As a result, finalised survey was reduced to only five questions. This reduced survey was estimated to be completed in under ten minutes, whereas the interview would only take a maximum of 45 minutes. This had the added benefit of ensuring a satisfactory response rate. 


\subsection{Participants}

An advertisement to recruit interested participants was disseminated in February 2021 through the use of official university e-mails and a social media application (WhatsApp). The survey itself was accessible through an online link, hosted on Google Forms. Interested participants were screened prior to starting the survey to ensure eligibility. The study information sheet and a consent and privacy notice was also made available to participants. Being a purposive sample, it was constantly ensured that participants from a variety of background were represented (faculty membership, academic performance, and prior statistics experience). This survey lasted for one month.

In total, 51 undergraduate students spanning across four main faculties (Arts, Business \& Economics, Health Sciences and Science) responded to the survey. However, unfortunately ten of these had to be discarded due to non-completion, leaving us with a total of $\mathrm{N}=41$ usable data points. Interviewees were recruited from the pool of respondents who agreed to be contacted. All the interviews were conducted in March 2021 in a span of 10 days.

\subsection{Data analysis}

In the descriptive summary of the data, mean scores and mean confidence ratings are segregated by each demographic variable. P-values (as reported in Table 3 g give an indication of whether the scores and confidence ratings are equivalent in the categories of each nominal demographic variable. This table therefore gives us, at a glance, several factors which may correlate with scores and/or confidence ratings.

To investigate the existence of misconception from the sample responses, a Chi-square test of independence was conducted on the two-by-two contingency table of correct item responses and confidence ratings. A proportion test was also carried out to compare the proportion of confident and non-confident responses from the sub-pool of incorrect answers. The idea here is that a rejection of the null in the Chi-square test and proportion test would indicate the presence of misconception. One weakness in performing the tests this way is that we have to assume that all test items are independent of each other. Although the test items for the most part are a measurement of different hypothesis test concepts, independence may not be a suitable assumption, since one respondent is responsible for five of the questions. Respondents which are more well-versed in statistics will surely tend to answer more of the survey items correctly with confidence, and vice versa. As a simple workaround, we also perform a statistical test using the total score and average confidence ratings for each respondent.

Due to nature of the data, the statistical tests mentioned above were conducted using methods which did not rely on normality or large-sample assumptions. Specifically, where Chi-square tests of independence were carried out on contingency tables with low cell counts, a Monte Carlo approximation to the p-values were obtained. Furthermore, tests of proportions were carried out using exact binomial tests, rather than relying on large-sample properties of the Z-test for proportions. Finally, the non-parametric Kruskal-Wallis test are employed in lieu of traditional analysis of variance (ANOVA).

Item difficulties (i.e., the proportion of times in which the survey questions were incorrectly answered) were calculated. This allowed us to identify the most misconceived knowledge domain of hypothesis testing, especially when taking the confidence ratings into account. In addition, we also focused on overall confidence ratings and per item, in an effort to see meaningful patterns of confidence, if any. Differences in confidence ratings among correct and incorrect answers were of particular interest.

All analyses were conducted using the open-source programming language R. A significance level of $10 \%$ was set for the statistical tests in our analyses. 


\section{Results}

In this section, we discuss the results of the quantitative and qualitative study. The obtained sample $(\mathrm{N}=41)$ was found to be a good representation of the student population of interest. Majority of the respondents $(53.5 \%)$ belonged to the Science faculty (home to the Mathematics Department), and a larger proportion of females responded compared to males (67.4\% vs. $32.6 \%$ ). An almost equal split of students were captured in terms of being in the final-year of study, being in a quantitative-based programme, and prior statistics experience. Finally, the distribution of the sample in terms of GPA does seem to be proportional to population based performance (academic bell curve), with the majority of students (79.1\%) sampled belonging to the medium GPA category.

\subsection{Quantitative analysis}

\subsubsection{Survey scores and confidence ratings}

Table 3 shows the demographic distribution of the sample, as well as survey scores and confidence ratings of each demographic section. In general, it was found that the overall performance on this survey was quite poor with a mean score of 1.37 in the sample $\left(1^{\text {st }}\right.$ Quartile $=0.00$; $3^{\text {rd }}$ Quartile $=2.00$ ). The lowest and highest score were 0 and 4 respectively, with $81.4 \%$ of respondents obtaining a score of two or lower, while $27.9 \%$ scored zero on this survey. Confidence ratings on the other hand are left-skewed (though the maximum range was observed) with a median confidence rating of seven. This suggests that respondents were largely confident of their answers. Mean scores were not found to be significantly different when segregated by gender ( $\mathrm{p}$ $=0.529$ ). Similarly, the mean scores across the four main faculties were also not found to be significantly different from one another $(\mathrm{p}=0.778)$. There does seem to be a slight disparity in confidence ratings by faculty membership $(\mathrm{p}=0.091)$, with Science students showing the highest confidence ratings and those from the Arts showing the lowest confidence ratings.

Students in their final year of study were expected to perform better on the survey compared to those not in their final year, since final-year students have more opportunities to encounter a statistics course during their term at university. Indeed, we see a significant difference of 0.7 points (at the $10 \%$ level) on average comparing the mean score of the two groups $(\mathrm{p}=0.066)$. Despite this fact, confidence ratings were not found to be different $(\mathrm{p}=0.283)$.

It was also expected that students taking a quantitative-based programme or students having prior statistics experience would score higher than their counterparts who do not, but the data seems to indicate that this is not the case $(\mathrm{p}=0.314$ and $\mathrm{p}=0.584$ respectively). Nonetheless, in both situations we found that students in quantitative-based programmes and with prior experience are more confident in their answers $(\mathrm{p}=0.054$ and $\mathrm{p}=0.014$ respectively). There is a slightly significant effect of GPA on mean scores $(\mathrm{p}=0.082)$, and that the data seems to show that students in the medium GPA bracket tend to score highest on average. There is no effect of GPA on confidence ratings $(\mathrm{p}=0.497)$, however.

\subsubsection{Identifying misconception}

To identify the presence of misconceptions, we differentiate the respondents based on their answers to the five survey items as per Table 2. Specifically, we constructed a $2 \times 2$ contingency table based on their answers (Right vs Wrong) as well as their confidence ratings (Confident vs Not confident) to each survey item. We are mainly interested in the pool of respondents who confidently produced incorrect answers. Figure 2 shows the frequency distribution of the responses based on the two categorical variables mentioned. There was a significantly higher proportion in this particular category than any of the three others $(\mathrm{p}=0.058)$.

However, it may be more appropriate to focus the analysis to the sub-sample with formal statistics training. Meaning to say, we now focus on the red bars in Figure 1. It is still found that 
Table 3: Demographic variables of the respondents, showing mean scores and confidence ratings in each demographic.

\begin{tabular}{|c|c|c|c|c|c|}
\hline \multirow[b]{2}{*}{ Variable } & \multirow[b]{2}{*}{ Count (\%) } & \multicolumn{2}{|c|}{ Score (out of 5 ) } & \multicolumn{2}{|c|}{ Confidence (out of 10 ) } \\
\hline & & Mean (SD) & p-value & Mean (SD) & p-value \\
\hline \multicolumn{6}{|l|}{ Gender } \\
\hline Female & $29(67.4 \%)$ & $1.28(1.03)$ & 0.529 & $5.80(1.97)$ & 0.707 \\
\hline Male & $14(32.6 \%)$ & $1.57(1.34)$ & & $5.81(1.39)$ & \\
\hline \multicolumn{6}{|l|}{ Faculty } \\
\hline Arts & $9(20.9 \%)$ & $1.00(0.87)$ & 0.778 & $4.62(2.50)$ & 0.091 \\
\hline Business \& Economics & $7(16.3 \%)$ & $1.57(1.51)$ & & $5.20(0.86)$ & \\
\hline Health Sciences & $4(9.3 \%)$ & $1.50(0.58)$ & & $5.75(1.45)$ & \\
\hline Sciences & $23(53.5 \%)$ & $1.43(1.20)$ & & $6.46(1.48)$ & \\
\hline \multicolumn{6}{|l|}{ In final year of study } \\
\hline Yes & $23(53.5 \%)$ & $1.70(1.29)$ & 0.066 & $6.19(1.40)$ & 0.283 \\
\hline No & $20(46.5 \%)$ & $1.00(0.79)$ & & $5.36(2.09)$ & \\
\hline \multicolumn{6}{|c|}{ Taking a quantitative-based programme } \\
\hline Yes & $20(46.5 \%)$ & $1.60(1.35)$ & 0.314 & $6.40(1.30)$ & 0.054 \\
\hline No & $23(53.5 \%)$ & $1.17(0.89)$ & & $5.29(2.00)$ & \\
\hline \multicolumn{6}{|c|}{ Prior statistics experience } \\
\hline Yes & $25(58.1 \%)$ & $1.48(1.29)$ & 0.584 & $6.42(1.43)$ & 0.014 \\
\hline No & $18(41.9 \%)$ & $1.22(0.88)$ & & $4.96(1.92)$ & \\
\hline \multicolumn{6}{|c|}{ Grade point average (GPA) } \\
\hline Low $(0.0-2.5)$ & $4(9.3 \%)$ & $0.50(1.00)$ & 0.082 & $6.70(1.79)$ & 0.497 \\
\hline Medium $(2.5-3.5)$ & $34(79.1 \%)$ & $1.56(1.08)$ & & $5.72(1.83)$ & \\
\hline High $(3.5-5.0)$ & $5(11.6 \%)$ & $0.80(1.30)$ & & $5.64(1.56)$ & \\
\hline
\end{tabular}




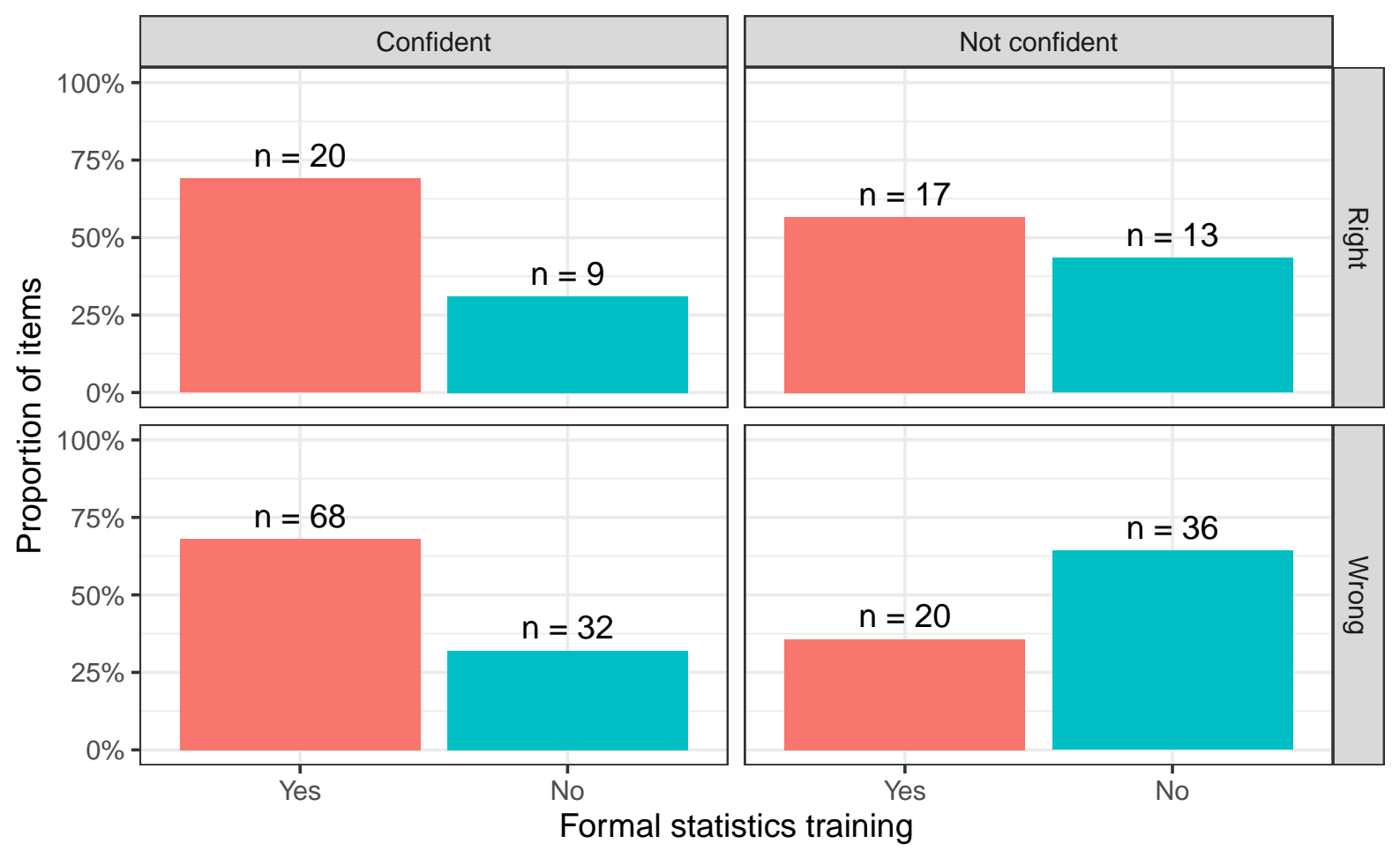

Figure 1: Cross-tabulation of survey item responses based on the two variables "Confident/Not confident" and the correctness of the item responses "Right/Wrong".

the category "Confident \& Wrong" has the highest value among the rest of the categories, and this cross tabulation was found to be significant at the $1 \%$ level $(\mathrm{p}=0.008)$. Further, comparing the two proportions of confident respondents and non-confident respondents, specifically among the pool of respondents with prior statistics experience who answered incorrectly, resulted in a strong rejection of the null $(\mathrm{p}<0.001)$. Repeating the above tests using mean scores and confidence ratings (i.e. averaged for each individual) yielded similar results ( $p=0.068$ for the Chi-square test of independence; $\mathrm{p}=0.096$ for the exact binomial test). Based on these results, we conclude that there is evidence of misconception regarding hypothesis testing procedures among students.

\subsubsection{Item analysis}

A frequency breakdown of the answer choices for each question is given in Table4. We observed some biases in answer choices, but only in Questions 1 and 3. The remaining questions did not show any statistically significant difference in regards to the spread of the answer choices.

The question with the most correct answers is Question 1 (Recognise Applicability), with $39.5 \%$ of respondents answering correctly. In fact, Question 1 was the only question where the most common answer was also the correct one. Interestingly, a similar knowledge domain question was posed in Question 2 and here most responses $(81.4 \%)$ were incorrect. The question with the highest item difficulty (most incorrect answers) was Question 3 (Generate Hypothesis), with only $14 \%$ identifying the correct answer. Looking at the distribution of confidence ratings in Figure 2, we can see that the median confidence rating among incorrect answers are much higher than among correct answers ( $\mathrm{p}=0.019$ using a Kruskal-Wallis test). When broken down by item, the same trend persists, although the difference in confidence ratings are only significant in item Question 5 ( $\mathrm{p}=0.036$ using a Kruskal-Wallis test). 
Table 4: Item analysis showing percentage breakdown of answer choices for each question in the survey. Bold options indicate correct answers

\begin{tabular}{|c|c|c|c|c|}
\hline Question & Category & Option & Count $(\%)$ & p-value \\
\hline 1 & Recognise Applicability & $\begin{array}{l}\mathrm{a} \\
\mathrm{b} \\
\mathrm{c} \\
\mathrm{d}\end{array}$ & $\begin{array}{r}17(39.5 \%) \\
7(16.3 \%) \\
6(14.0 \%) \\
13(30.2 \%)\end{array}$ & 0.059 \\
\hline 2 & Recognise Applicability & $\begin{array}{l}a \\
b \\
c \\
d\end{array}$ & $\begin{array}{r}8(\mathbf{1 8 . 6 \% )} \\
11(25.6 \%) \\
16(37.2 \%) \\
8(18.6 \%)\end{array}$ & 0.291 \\
\hline 3 & Generate Hypothesis & $\begin{array}{l}a \\
b \\
c \\
d\end{array}$ & $\begin{array}{r}14(32.5 \%) \\
20(46.5 \%) \\
6(\mathbf{1 4 . 0 \% )} \\
\quad 3(7.0 \%)\end{array}$ & $<0.001$ \\
\hline 4 & Conclusion & $\begin{array}{l}a \\
b \\
c \\
d\end{array}$ & $\begin{array}{r}8(18.6 \%) \\
12(27.9 \%) \\
\mathbf{1 5}(\mathbf{3 4 . 9 \% )}) \\
8(18.6 \%)\end{array}$ & 0.383 \\
\hline 5 & Decision Rule & $\begin{array}{l}\mathrm{a} \\
\mathrm{b} \\
\mathrm{c} \\
\mathrm{d}\end{array}$ & $\begin{array}{r}12(27.9 \%) \\
5(11.6 \%) \\
\mathbf{1 3}(\mathbf{3 0 . 2 \%}) \\
13(30.2 \%)\end{array}$ & 0.246 \\
\hline
\end{tabular}
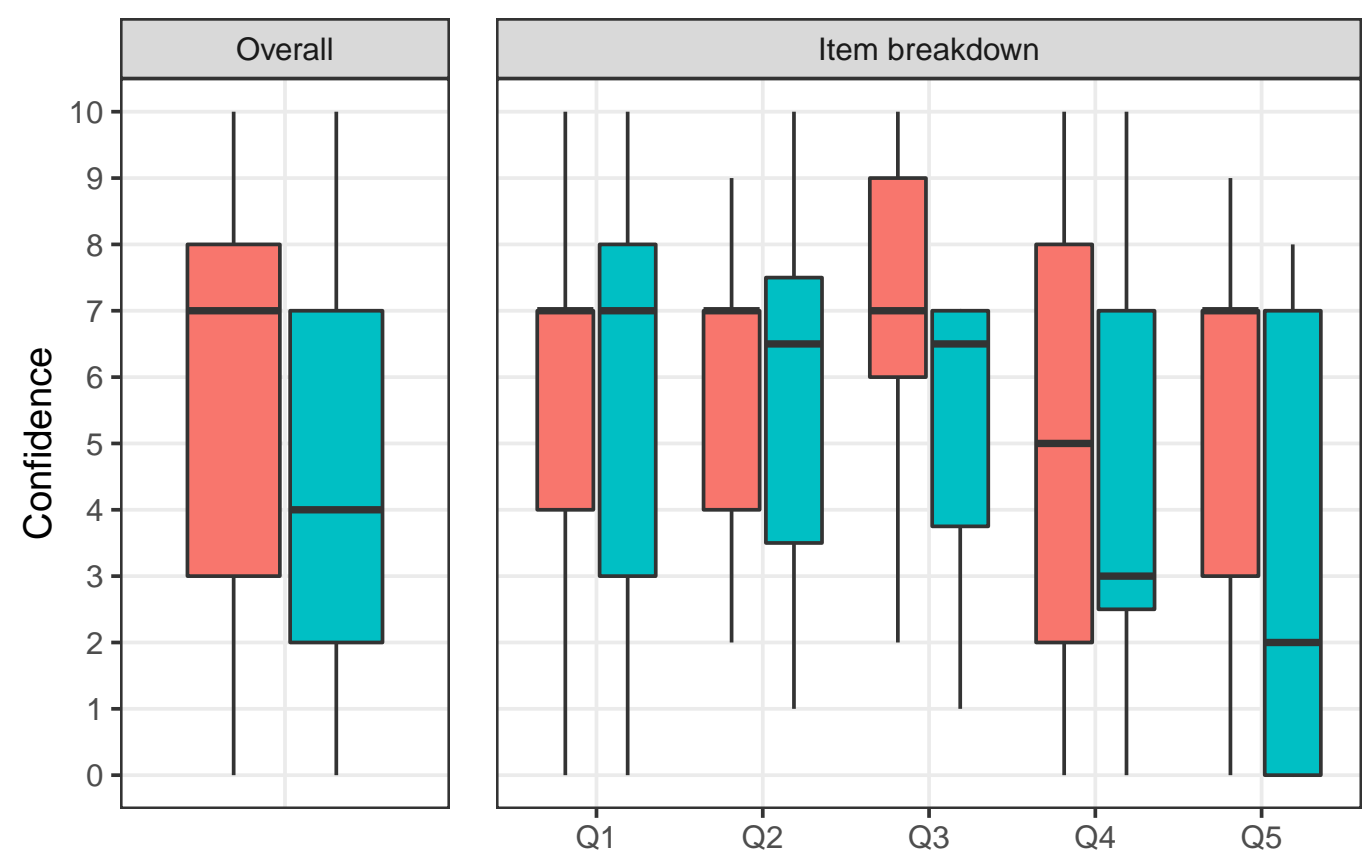

Answers

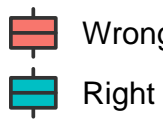

Figure 2: Distribution of overall confidence ratings (for all items) as well as a broken down by item. 


\subsection{Qualitative analysis}

This section presents the analysis from the follow-up interviews to qualitatively examine indepth the common errors made by undergraduate students. The analyses will provide insights of students' understanding of hypothesis question. For each study item, excerpts from transcribed interviews are included in the appendix. Five interviewees were available for interview, and Table 5 summarises their characteristics.

Table 5: Charateristics of interviewees. Correct answers are shown in bold.

\begin{tabular}{rlllllc}
\hline No. & Gender & Major & $\begin{array}{l}\text { Final- } \\
\text { year }\end{array}$ & Statistical experience & $\begin{array}{c}\text { Answers to } \\
\text { survey }\end{array}$ & Score \\
\hline 7 & Female & Mathematics & Yes & Prior \& during university & a, c, b, a, c & 2 \\
14 & Male & Mathematics & Yes & Prior \& during university & a, a, b, c, a & 3 \\
19 & Female & Mathematics & Yes & Only during university & d, a, c, b, c & 3 \\
20 & Female & Biology & Yes & Prior to university & d, d, b, d, d & 0 \\
28 & Female & Mathematics & No & Prior \& during university & b, d, b, b, b & 0 \\
\hline
\end{tabular}

\subsubsection{Recognising applicability}

Questions 1 and 2 test students' ability to recognize the applicability of hypothesis testing. A majority $(39.5 \%)$ of the respondents answered Question 1 correctly (option a). From this it is discerned that in general students do understand that one of the goals of hypothesis testing is to be able to compare statistics of interest (most commonly the mean) between two groups. In fact, Interviewee 14 arrived at the correct choice by a process of elimination, arguing that there is a want to compare two groups. Of the five interviewees, two chose the correct option a, while the remaining chose between options $\mathrm{b}$ and $\mathrm{d}$.

1. Which of the following is most likely to be answered by a study that requires statistical hypothesis testing?

a. Do athletes have a lower GPA than other students? [39.5\%]

b. What equation predicts a student's GPA from his/her IQ? [16.3\%]

c. What are the typical costs for full-time university students in Brunei? [14.0\%]

d. Do the 11:50am Korean language students perform better than the 2:00pm Korean language students this semester? [30.2\%]

Figure 3: Item 1 on Recognise Applicability. Correct answer shown in bold, and percentage responses for each option shown in square parentheses.

Options b and $\mathrm{c}$ are distractor options which are not at all relevant to hypothesis testing. In the interviews, students gave similar explanations for discounting options b and c, stating that these two options were not making a comparison between two groups.

The most common wrong choice was option $\mathrm{d}(30.2 \%)$, which implies that students get confused between the concepts of 'population' and 'sample'. This is because option d itself can be answered without hypothesis testing as the population is known to be (or at least assumed to be) finite and obtainable. Small populations do not require sampling and hence, statements can be directly made from the mean of both groups. Most of the interviewees admit however that they were confused between this option and the correct choice. 
Interestingly, interviewees who answered d (interviewees 19 and 20) mentioned that the stated times made option d more specific in nature, and in a manner, contained "more data". They wrongly concluded that highly specific answers made for a more plausible answer, and thus seemingly made choices from a position of ignorance. Interviewee 7 (who answered correctly) on the other hand thought that the stated times are not of significance, yet insisted on switching their choice to d because "choice a was too broad".

2. Which of the following statements is the best justification for using a statistical hypothesis test to answer the question: Are female students as successful as male students in college mathematics?

\section{a. It allows you to talk about uncertainty associated with your decision. [18.6\%]}

b. It allows you to use only a sample of students to prove something for all students. [25.6\%]

c. It allows you to calculate means and use statistical tables to answer the questions. $[37.2 \%]$

d. It allows you to find and prove the answer using mathematical calculation. [18.6\%]

Figure 4: Item 2 on Recognise Applicability. Correct answer shown in bold, and percentage responses for each option shown in square parentheses.

We now discuss Question 2. With a similar objective to Question 1, surprisingly the correct choice (option a) was chosen the least frequently (18.6\%) among respondents. Besides recognising applicability, this question aimed to assess whether students understand the role of probability and uncertainty associated with decision-making in hypothesis testing. Two interviewees (14 and 19) originally chose the correct answer, and after some delibaration, Interviewee 28 changed course to option a as well. However, their reasons for choosing the correct answer were not terribly convincing. Circular reasoning were provided, such as "I'm choosing option a because that's the procedure of statistical hypothesis testing" and "Option a has the steps to get [sic] hypothesis test".

The most popular choice was option c $(37.2 \%)$, followed by option b (25.6\%). While option $\mathrm{c}$ is true, it is not the best justification among the four choices. Option b offers, according to Smith (2008), a view that is often misconstrued, which is the notion of a sample proving a statement for population. None of the interviewees answered b.

Some interviewees (Interviewee 20 and 28) thought that statistical hypothesis testing strictly concerns procedures that are mathematical and deterministic in nature (option d). Interviewee 20 actually had second thoughts about their answer, and prompted the interviewer for the definition of hypothesis testing before deciding to switch answers to option b, which was still incorrect.

To summarise, it was recognised in general that statistical hypothesis testing is useful for making comparisons between two groups. However, students did not understand that hypothesis testing is used for inference to a wider population. This result was similar to Smith's (2008) results: "They did not mention the role of inference in statistical hypothesis testing. They did not mention that statistical hypothesis tests provide a means of dealing with the variability that exists between and among samples.". Furthermore, students fail to recognise the principle of randomness associated with sampling and inference. A dire misconception regarding the deterministic nature of hypothesis test procedures was also held. 


\subsubsection{Generating hypothesis}

Question 3 tests students' ability to formulate hypothesis. In this case, since the effect to be proven is that students have higher GPA than science students on average (the alternative hypothesis), the correct null hypothesis is the complement of this statement, i.e. the mean GPA of humanities students is less than or equal to that of science students (option c). Only $14.0 \%$ of respondents answered this correctly. It would be understandable if the correct answer was confused with option d, i.e. a similar statement to option $\mathrm{c}$ without the equality statement. However, an even lower proportion of students $(7.0 \%)$ chose this option. There really seems to be a strong misconception regarding formulating hypotheses.

3. A researcher would like to establish that humanities students have higher GPA than science students. Which of the following null hypotheses should be tested? The mean GPA of humanities students is:

a. greater than that of science students. [32.6\%]

b. greater than or equal to that of science students. [46.5\%]

c. less than or equal to that of science students. [14.0\%]

d. less than that of science students. [7.0\%]

Figure 5: Item 3 on Generate Hypothesis. Correct answer shown in bold, and percentage responses for each option shown in square parentheses.

Among the interviewees, only one chose the correct answer (option c), while the remaining four opted for option b. Interestingly, when given the chance to switch their answers, everyone chose from option a or b, even the interviewee with the correct answer initially. For instance, Interviewee 7, who decided upon option a (after switching from b), gives their explanation as follows:

Interviewee 7: I think I'm changing it to a. For the question, the humanities have higher GPA than science students so that means... the means of GPA should be more than the science students which eliminates $\mathrm{c}$ and $\mathrm{d}$ and then difference equals to... [inaudible] that means the GPA is equal does not mean humanities has higher GPA, so a.

The excerpt above suggests that the students is actually reasoning about the alternative hypothesis (the effect that is hoped to be proven) instead of the null hypothesis. Interestingly, the rest of the interviewees reason along similar lines. Perhaps students' error to this question can be attributed to a 'labelling' confusion about the two kinds of hypotheses. However, those who answered option b were adamant that the null hypothesis must contain equality. This kind of reasoning on the part of students does not fit in with a simple null/alternative hypothesis confusion. While some of the interviewees do acknowledge that the null and alternative hypotheses should contradict each other, there does not seem to be a clear understanding on the part of students regarding what effect is wished to be proven (or disproven).

\subsubsection{Test conclusion}

Question 4 tests students' ability to make the correct conclusive statements after a hypothesis test is conducted. In particular, students were asked to select the statement which gives the best interpretation of the sample statistics. Overall, answer choices were pretty uniformly distributed. The correct choice (option c) was chosen most frequently (34.9\%), and this is followed by option b (27.9\%). 
Most surprisingly, interviewees expressed a clear understanding of the difference between sample and population, and how statistics derived from a sample do not equate necessarily to statistics of the population. Interviewee 7 even showed understanding of sampling variability, reasoning "for this sample it could be like this, but for another sample it could be different". Interviewee 14 eliminated option a because while being true, "it has nothing to do with inference". Remarkably, almost none could not reason in this manner in the previous three questions. Perhaps one of the factors for this collective epiphany is the sentencing of the question itself, with keywords such as 'information from samples' and 'inference' present. Indeed, if one truly understood the concept of sampling from a population, one would eliminate options b and $d$ totally.

4. A study tested the claim that: 'Transfer students are less successful than students admitted as first-time freshmen'. Results showed a difference in first semester GPA that is significant at the 0.05 level. Information from samples is shown below. What is the most reasonable inference about the population of all first semester students that can be drawn from this information?

\begin{tabular}{lcc}
\hline & Transfer Admits & Freshman Admits \\
\hline$n$ & 50 & 50 \\
mean GPA & 2.5 & 2.8 \\
\hline
\end{tabular}

a. There are equal numbers of transfer and first-time freshman students. [18.6\%]

b. The mean first semester GPA of all freshman admits is 0.3 greater than that of all transfer admits. [27.9\%]

c. It is unlikely that the first semester GPA of all transfer admits equals that of all freshman admits. [34.9\%]

d. The mean first semester GPA of all university students is $(2.5+2.8) / 2$ or about 2.65 . $[18.6 \%]$

Figure 6: Item 4 on Test Conclusion. Correct answer shown in bold, and percentage responses for each option shown in square parentheses.

Students who did choose option b or d over the correct choice of $\mathrm{c}$ argued that it was the vagueness of the statement in option $c$ which swayed them to choose $b$. Meaning to say, the fact that a 0.3 difference was specified in option b made for a more compelling choice. One even asserted that option d was factual, and hence was most confident in that choice.

The interviewee who correctly answered this question explained that they were looking for phrases such as 'significance level' and 'p-values' in any of the the answer choices, indicating that they understood that the question was looking for a conclusion to some hypothesis test. This was not an acceptable reason for the answer, however.

In summary, it seems evident that a majority of the interviewees do not have a deep understanding of hypothesis testing as an inferential tool. While discussion did revolve briefly about concepts of uncertainty and variability in sampling, these were quite superficial.

\subsubsection{Decision rule}

Question 5 tests the understanding of 'significance level', and its part in concluding a hypothesis test. The almost uniform spread of the answer choices does suggest a poor understanding of the topic. In order to arrive at the correct answer, students must simultaneously know the 
definition of significance levels (or equivalently the concept of Type I error), and appreciate the effect that a higher significance level has on the outcome of a test. Two students managed to arrive at the correct choice.

Interviewees were asked to define 'significance level'. Some interviewees did manage to explain the concept correctly by sketching a bell curve with rejection regions on both side of the tails. Those who were not able to were found to possess another common misconception that null hypotheses may be accepted as true (if there is not enough evidence to reject it). Some admitted not knowing what alpha values are used for. Interestingly, one interviewee confused significance level with 'confidence level', i.e. the complementary concept associated with confidence intervals. Thus, incorrect answers to this item stems from a lack of understanding on the definition of significance level and Type I errors, rather than a misconception per se.

5. To test the hypothesis that private schools are more effective than public schools, researchers plan to compare the mean starting salaries of private and public school graduates. But they cannot agree on whether to test the results at a significance level of 0.05 $(\alpha=0.05)$ or at a significance level of $0.10(\alpha=0.10)$. What effect will using 0.10 rather than 0.05 have on the study?

a. Using 0.10 will result in a greater chance that they will incorrectly retain the hypothesis. [27.9\%]

b. Using 0.10 will result in a greater chance that the null hypothesis is actually true. $[11.6 \%]$

c. Using 0.10 will result in a greater chance that they will incorrectly reject the null hypothesis. [30.2\%]

d. Using 0.10 will result in a greater chance that the alternative hypothesis is actually true. $[30.2 \%]$

Figure 7: Item 5 on Decision Rule. Correct answer shown in bold, and percentage responses for each option shown in square parentheses.

\section{Discussion and conclusion}

This study aimed to investigate undergraduate students' misconception of hypothesis test concepts. To this end, a two-phase quantitative and qualitative survey was conducted at Universiti Brunei Darussalam. The major finding of the study was the presence of misconceptions across several hypothesis test concepts among undergraduate students, as indicated by the low scores yet high confidence ratings in the responses to the questionnaires.

There was no significant difference in scores found among students from various faculties and programmes. Despite this, quantitative and science students tend to be more confident in their answers. While there is a small association between GPA grades and survey score, it was found that students in the Medium GPA bracket tend to score the highest. All of this is more or less in agreement with findings from previous studies, that high academic performance do not necessarily correlate with having perfect understanding of statistical concepts (Clark et al., 2007; Mathews \& Clark, 2003; Smith, 2008). Based on the item difficulties to our survey items, students face the most difficulties in the following areas: Generating hypotheses, understanding significance levels, and understanding the concept of a hypothesis test. The latter two are in line with findings from numerous studies in this area (Batanero, 2000, del Mas et al., 2007; Haller \& Krauss, 2002; Vallecillos, 2000; Williams, 1998). 
It was often thought that statements from samples extend directly (without error) to that of the population. Some even hold the belief that hypothesis testing offers a mathematical formula to directly proof or disprove hypotheses. A persistent belief is held among students students that hypothesis testing is strictly procedural in nature, and simply following a mechanistic framework allows one to achieve the desired objective of proving or disproving a hypothesis. These same misconceptions were also addressed in Smith (2008) and Sotos et al. (2009). For instance, Sotos et al. (2009) stated that "... the two major misconceptions for the concept of hypothesis test were just as popular among our students: $20 \%$ of them believed that a hypothesis test is a mathematical (logical) proof of the null hypothesis".

Our study also highlights the issue of generating hypotheses among students, which has not received much attention thus far in previous studies. Students greatly confuse what exactly constitutes a 'null' hypothesis. In Question 3 of the survey, students were asked to select the correct null hypothesis in a study wishing to prove that humanities students have higher GPA than science students. A majority of the students wrongly equated the null hypothesis directly with the statement 'humanities students have higher GPA than science students'. There could have been a simple confusion about exchanging the null/alternative hypothesis, but the interviews certainly suggest otherwise.

Switching attention to confidence of students in their answers, the data suggests the presence of a negative association between correct answers and confidence in answers. This is in contrast to some prior studies done in this regard. Allen et al. (2006) and Sotos et al. $(\overline{2009})$ found a positive trend, while Chance et al. (2004) found no suggestive relationships at all. When looking at it from the perspective of our proposed misconception framework (see Table 2), the results here reinforces the notion that students have a distorted understanding of hypothesis testing concepts, yet are confident in their misunderstandings, demonstrating existence of misconceptions among students. On a per item basis, the same negative trend persists but were not found to be significant except for Question 5 (on decision rule and significance levels).

In light of the responses to the survey and interviews, it is important for educators to understand potential sources of misconception. One of the most alarming aspects of the interview was how weak students' line of reasoning were in selecting their answers, revealing an overall poor understanding of inferential statistics. We have seen that students across various backgrounds are prone to suffering from misconceptions, which suggests some form of commonality in teaching methods across disciplines. Indeed, misconceptions among statistics students are well known (Sotos et al., 2007) and a reform in statistics education has even taken place. This reform hopes to promote more of statistical reasoning, thinking, and literacy (Ben-Zvi \& Garfield, 2004) something that we concur is lacking among the present cohort of students in our study. With the rise of cross-displicinary quantitative programmes such as psychometrics, systems biology, educational testing, and so on, the need for practising statisticians in their own field is more than ever. In such programmes however, the learning of statistical methods is often concentrated to applied situations (use of software, obtaining p-values for reporting, etc.), and thus the appreciation for the mathematical finer details is lost. This is by no means a call for such programmes to integrate deeply advanced mathematics and probability into such applied modules, but certainly a balance must be struck, otherwise more and more graduates with wrongly conceived notions about hypothesis testing and statistics in general will be generated.

In our view, apprehension towards statistical studies is widespread, which may have been a factor in the low response rates to our survey. No formal study has yet to be conducted regarding anxiety in statistics (Hanna et al., 2008) in the South East Asian region including Brunei Darussalam, as far as we know, but if found to exist then this could affect student outcomes. For instance, through a mediation model, Primi et al. (2018) show that statistics anxiety mediates the relationship between probabilistic reasoning ability and achievement outcomes. In such a case, monitoring of students' attitudes and beliefs during their statistics education experience warrants more attention, as doing so can lead to less frustrations and more effective learning of 
statistics (Gal et al., 1997).

From experience, the topic of hypothesis tests can be quite a dry subject, challenging even the most attentive of students. Here, we give some suggestions to improve the teaching of statistics course based on the three pillars of content, pedagogy and technology (Moore, 1997).

- Break away from the traditional chalk-and-talk style of teaching. Students also learn better if they are engaged in and are motivated to struggle with their own learning. Research suggests that working cooperatively in small groups is beneficial to solve problems and learn to argue convincingly (Chick \& Watson, 2002). Another suggestion is the use of concept cartoons (Keogh \& Naylor, 1999) which helps formulate ideas and concepts explicitly and promotes class discussions.

- Technology (computers, graphic calculators, software, internet) can be used to promote statistical learning. Studies have shown that a well-designed simulation can play a significant role in enhancing students' ability to grasp statistical concepts and random processes (Lane \& Tang, 2000; Mills, 2004). Some of these techniques can be integrated easily and successfully into an instructor's syllabus, especially in a mixed-method approach coupled with the use of a learning management system (also known as blended learning - see Jamil et al., 2021).

- For the interested reader, a great introduction to the concept of p-values is given in a blog post and accompanying video by Kozyrkov (2021) where the concept is introduced as the amount of 'surprise factor' upon inspecting the evidence, given some preconceived notion of the universe. That it is whimsically explained with the help of charming puppies does add to the appeal and helps combat the dry nature of the topic. Informal ideas can help build formal ideas (Schwartz et al., 2007). Engage students' prior knowledge to help motivate and develop formal reasoning about statistical concepts.

Our investigation into specific factors which affect misconceptions in statistics did not yield satisfactory insights. Besides the Kruskal-Wallis test comparing scores in various demographic groups, attempts at fitting a regression model to explain variation in misconceptions using available independent variables was done to very little success. Perhaps our choice of using GPA and prior statistics experience (having taken any statistics class formally) was a poor one. We expected that these variables can act as proxies for actual 'statistics performance', but this may not have been the case. An improvement to the study would be to design the study around students taking a statistics class at university, although this would perhaps require sampling from multiple cohorts in different years to investigate persistence of misconceptions.

We also unexpectedly found hesitance on respondents' part to reveal their exact GPA, and this resulted in incomplete responses which had to be discarded. In hindsight, asking for GPA intervals would have been a better thing to do. On this note, perhaps several other factors may also affect response and completion rates which need to be studied, which may also be linked to a study on statistics anxiety mentioned above.

The use of confidence ratings in a two-tier questionnaire is not common, and in fact, literature surrounding its use is sparse. Typically, confidence rating data are collected to study relationships between confidence and misconception, which was not the main motivation of this study. Whether or not the usual two-tier format (asking students' for their reasons) is a better option remains to be investigated. Theoretical foundations for our misconception categorisation (Table 2) warrants further study as well. 


\section{References}

Allen, K., Rhoads, T. R., \& Terry, R. (2006). Work in Progress: Assessing Student Confidence of Introductory Statistics Concepts. Proceedings. Frontiers in Education. 36th Annual Conference, 13-14. https://doi.org/10.1109/fie.2006.322590

Arslan, H. O., Cigdemoglu, C., \& Moseley, C. (2012). A Three-Tier Diagnostic Test to Assess Pre-Service Teachers' Misconceptions about Global Warming, Greenhouse Effect, Ozone Layer Depletion, and Acid Rain. International Journal of Science Education, 34(11), 1667-1686. https://doi.org/10.1080/09500693.2012.680618

Bandura, A. (1977). Self-efficacy: toward a unifying theory of behavioral change. Psychological review, $84(2), 191$.

Batanero, C. (2000). Controversies Around the Role of Statistical Tests in Experimental Research. Mathematical Thinking and Learning, 2(1-2), 75-97. https://doi.org/10.1207/ S15327833MTL0202_4

Ben-Zvi, D., \& Garfield, J. (2004). The Challenge of Developing Statistical Literacy, Reasoning and Thinking. https://doi.org/10.1007/1-4020-2278-6

Burns-Childers, A., Rock, L., Chamberlain, D., Kemp, A., Meadows, L., Stalvey, H., \& Vidakovic, D. (2017). Students' Understanding of Test Statistics in Hypothesis Testing. Proceedings of the 20th annual conference on research in undergraduate mathematics education (pp. 82-92).

Cambridge Assessment International Education. (2018). Cambridge O Level Statistics 4040 Syllabus: For examination in November 2021. https://www.cambridgeinternational. org/Images/502923-2021-syllabus-.pdf

Cambridge Assessment International Education. (2020). Cambridge International AS \& A Level Mathematics 9709. https:/ / www. cambridgeinternational.org/Images / 415060- 20202022-syllabus.pdf

Chance, B., Mas, R. d., \& Garfield, J. (2004). Reasoning about Sampling Distribitions. In D. Ben-Zvi \& J. Garfield (Eds.), The challenge of developing statistical literacy, reasoning and thinking (pp. 295-323). https://doi.org/10.1007/1-4020-2278-6_13

Chick, H. L., \& Watson, J. M. (2002). Collaborative influences on emergent statistical thinking - a case study. The Journal of Mathematical Behavior, 21(3), 371-400. https://doi. org/10.1016/s0732-3123(02)00135-9

Clark, J., Kraut, G., Mathews, D., \& Wimbish, J. (2007). The fundamental theorem of statistics: Classifying student understanding of basic statistical concepts. Unpublished manuscript. http://www1.hollins.edu/faculty/clarkjm/stat2c.pdf

del Mas, R., Garfield, J., Ooms, A., \& Chance, B. (2007). Assessing students' conceptual understanding after a first course in statistics. Statistics Education Research Journal, 6(2), $28-58$.

Gal, I., Ginsburg, L., \& Schau, C. (1997). Monitoring attitudes and beliefs in statistics education. In I. Gal \& J. B. Garfield (Eds.), the assessment challenge in statistics education.

Gurel, D. K., Eryılmaz, A., \& McDermott, L. C. (2015). A review and comparison of diagnostic instruments to identify students' misconceptions in science. Eurasia Journal of Mathematics, Science \& Technology Education, 11(5), 989-1008.

Haller, H., \& Krauss, S. (2002). Misinterpretations of significance: A problem students share with their teachers. Methods of Psychological Research, 7(1), 1-20.

Hanna, D., Shevlin, M., \& Dempster, M. (2008). The structure of the statistics anxiety rating scale: A confirmatory factor analysis using UK psychology students. Personality and Individual Differences, 45(1), 68-74. https://doi.org/10.1016/j.paid.2008.02.021

Ismail, Z., \& Chan, S. W. (2015). Malaysian students' misconceptions about measures of central tendency: An error analysis. AIP Conference Proceedings, 1643(1), 93-100. https://doi. org $/ 10.1063 / 1.4907430$ 
Jamil, H., Ramli, H. M., \& Leong, E. (2021). Advocating Blended Learning for University Undergraduate Level Mathematical Instruction Beyond Covid-19. In S. A. Abdul Karim \& S. A. Husain (Eds.), Engineering and Sciences Teaching and Learning Activities: New Systems Throughout COVID-19 Pandemics (pp. 33-45). Springer. https://doi.org/10. 1007/978-3-030-79614-3_4

Johnson, R. B., \& Onwuegbuzie, A. J. (2004). Mixed Methods Research: A Research Paradigm Whose Time Has Come. Educational Researcher, 33(7), 14-26. https://doi.org/10. 3102/0013189x033007014

Kaplan, J. J. (2009). Effect of Belief Bias on the Development of Undergraduate Students' Reasoning about Inference. Journal of Statistics Education, 17(1). https://doi.org/10. 1080/10691898.2009.11889501

Keogh, B., \& Naylor, S. (1999). Concept cartoons, teaching and learning in science: an evaluation. International Journal of Science Education, 21(4), 431-446. https://doi.org/10. 1080/095006999290642

Kirbulut, Z. D., \& Geban, O. (2014). Using three-tier diagnostic test to assess students' misconceptions of states of matter. Eurasia Journal of Mathematics, Science and Technology Education, 10(5), 509-521.

Kozyrkov, C. (2021). Explaining p-values with puppies. https://hackernoon.com/explaining-pvalues-with-puppies-af63d68005d0

Krishnan, S., \& Idris, N. (2014). Students' Misconceptions about Hypothesis Test. Journal of Research in Mathematics Education, 3(3), 276-293. https://doi.org/10.4471/redimat. 2014.54

Lane, D. M., \& Tang, Z. (2000). Effectiveness of Simulation Training on Transfer of Statistical Concepts. Journal of Educational Computing Research, 22(4), 383-396. https://doi. org/10.2190/w9gw-5m9c-uqvt-1e0r

Lee, C., \& Meletiou, M. (2003). Some difficulties of learning histograms in introductory statistics. Joint Statistical Meetings-Section on Statistical Education.

Liu, Y. (2005). Teachers' understandings of probability and statistical inference and their implications for professional development (Doctoral dissertation). https://ir.vanderbilt.edu/ handle/1803/13009

Mathews, D., \& Clark, J. (2003). Successful students' conceptions of mean, standard deviation, and the Central Limit Theorem. Unpublished paper. Retrieved 20 October $200 \%$.

Mevarech, Z. R. (1983). A deep structure model of students' statistical misconceptions. Educational Studies in Mathematics, 14(4), 415-429. https://doi.org/10.1007/bf00368237

Mills, J. D. (2004). Learning Abstract Statistics Concepts Using Simulation. Educational Research Quarterly, 28(4), 18-33.

Moore, D. S. (1997). New Pedagogy and New Content: The Case of Statistics. International Statistical Review, 65 (2), 123-137. https://doi.org/10.1111/j.1751-5823.1997.tb00390.x

Nickerson, R. S. (2000). Null hypothesis significance testing: A review of an old and continuing controversy. Psychological Methods, 5(2), 241.

Osborne, R. J., \& Gilbert, J. K. (1980). A technique for exploring students' views of the world. Physics Education, 15(6), 376. https://doi.org/10.1088/0031-9120/15/6/312

Primi, C., Donati, M. A., \& Chiesi, F. (2018). Teaching and Learning Stochastics, Advances in Probability Education Research. ICME-13 Monographs, 145-157. https://doi.org/10. 1007/978-3-319-72871-1_9

Rossman, A. J., \& Chance, B. L. (2014). Using simulation-based inference for learning introductory statistics. Wiley Interdisciplinary Reviews: Computational Statistics, 6(4), 211221. https://doi.org/10.1002/wics.1302

Schwartz, D. L., Sears, D., \& Chang, J. (2007). Reconsidering Prior Knowledge. In M. Lovett \& P. Shah (Eds.), Thinking with data (pp. 319-344). Psychology Press. 
Sekretariat Tetap Wawasan Brunei 2035. (2020). WAWASAN 2035 wawasan negaraku. Dewan Bahasa dan Pustaka, Kementerian Kebudayaan, Belia dan Sukan. http://www. wawasanbrunei.gov.bn/

Smith, T. M. (2008). An investigation into student understanding of statistical hypothesis testing (Doctoral dissertation). University of Maryland, College Park.

Sotos, A. E. C., Vanhoof, S., Noortgate, W. V. d., \& Onghena, P. (2007). Students' misconceptions of statistical inference: A review of the empirical evidence from research on statistics education. Educational Research Review, 2(2), 98-113. https://doi.org/10. 1016/j.edurev.2007.04.001

Sotos, A. E. C., Vanhoof, S., Noortgate, W. V. d., \& Onghena, P. (2009). How Confident are Students in their Misconceptions about Hypothesis Tests? Journal of Statistics Education, 17(2). https://doi.org/10.1080/10691898.2009.11889514

Treagust, D. F. (1988). Development and use of diagnostic tests to evaluate students' misconceptions in science. International Journal of Science Education, 10(2), 159-169. https: //doi.org/10.1080/0950069880100204

Vallecillos, A., \& Batanero, C. (1997). Activated concepts in the statistical hypotheses contrast and their understanding by university students. Recherces en Didactique des Mathematiques, 17, 29-48.

Vallecillos, A. (2000). Understanding of the Logic of Hypothesis Testing Amongst University Students. Journal für Mathematik-Didaktik, 21(2), 101-123. https://doi.org/10.1007/ bf03338912

Williams, A. M. (1998). Students' understanding of the significance level concept. Proceedings of the 5th International Conference on Teaching Statistics. https://iase-web.org/ documents/papers/icots5/Topic6p.pdf?1402524957 